\title{
Analyticity of Semigroups generated by Degenerate Mixed Differential Operators
}

\author{
Adel Saddi \\ Department of Mathematics, College of Education for Girls, King Khalid University, Abha, Saudi Arabia \\ E-mail: adel.saddi@fsg.rnu.tn \\ Received January 18, 2011; revised March 14, 2011; accepted March 20, 2011
}

\begin{abstract}
In this paper we are interested in studying the dissipativity of degenerate mixed differential operators involving an interface point. We show that, under particular interface conditions, such operators generate analytic semigroups on an appropriate Hilbert space $\mathrm{H}$. To illustrate the results an example is discussed.
\end{abstract}

Keywords: Adjoint, Interface, Dissipative Operators, Analytic Semigroups

\section{Introduction}

The evolution of a physical system in time is usually described in a Banach space by an initial value problem for a differential equation on the form:

$$
\left\{\begin{array}{c}
\frac{\mathrm{d} U(t)}{\mathrm{d} t}+L U(t)=0, \quad t \geq 0 \\
U(0)=U_{0}
\end{array}\right.
$$

Such problems are well posed in Banach space $X$ if and only if the operator $L$ generates a $C_{0}$-semigroup $\left(T_{t}\right)_{t>0}$ on $X$ [1]. Here the solution $U(t)$ is given by $U(t)=T_{t} U_{0}$ for $U_{0} \in D(L)$.

Problems involving interface arise naturally in many applied situation such as acoustic wave in ocean [2] and also as heat conduction in non homogeneous bodies. A systematic study of interface problems involving ordinary differential operator was done in [3].

Several authors have been interested to differential operators with matrix coefficients. Such operators arise in diverse range of applications (e.g. in Quantum physics), some examples in harmonic analysis have been treated in [4-6] and for an example in semigroups theory we refer to [7-8].

In this paper, inspired in the works of A. Saddi and O. A. Mahmoud Sid Ahmed [9] and also that of T. G. Bhaskar and R. Kumar [10], we establish with suitable assumptions the analyticity of semigroups generated by a class of differential operators involving matching interface conditions in the setting of complex Hilbert space.

As it is well known, in order that an operator $L$ gen- erates an analytic semigroup it suffices that it satisfies the $m$-dissipativity and we must have (see [11])

$$
\mathfrak{R e}\langle L U, U\rangle+\delta|\mathfrak{I} m\langle L U, U\rangle| \leq 0, \delta>0
$$

The paper is organized as follows: In section 2 we introduce the different notions and notations which we shall need in the sequel. In section 3 we study the mixed operator $L$ and its adjoint $L^{*}$ and we investigate some of its properties. In section 4 we study the dissipativity of the operator $(L-\lambda)$ and its adjoint for some suitable real number $\lambda$. We show that, under particular interface conditions, such operators generate strongly continuous semigroups. Using the previous results we conclude in section 5 with the aim of the paper about generation of analytic semigroups of operators with respect some regular interface conditions. Finally we discuss an example as an application to our results.

\section{Notations and Preliminaries}

Let $M_{n}(\mathrm{C})$ be the space of all square $n$ order matrix with complex coefficients, and $G L_{n}(\mathrm{C})$ the subset of $M_{n}$ (C) consisting of invertible matrices. The adjoint of a matrix $\boldsymbol{A} \in M_{n}(\mathrm{C})$ is denoted by $\boldsymbol{A}^{*}$.

Let $I_{1}=[a, 0], I_{2}=[0, b]$, where $-\infty<a<0<b$ $<+\infty$, and $I_{k}^{*}=I_{k} \backslash\{0\}$. For $k=1,2$ and an interval $X_{k} \subseteq I_{k}$, denote by $L^{2}\left(X_{k}, \mathrm{C}\right)$ the complex Hilbert space defined by

$$
L^{2}\left(X_{k}, \mathrm{C}\right)=\left\{u: X_{k} \rightarrow \text { C, measurable } / \int_{X_{k}}|u(t)|^{2} \mathrm{~d} t<+\infty\right\}
$$

endowed with the canonical inner product 


$$
\langle u, v\rangle_{k}=\int_{X_{k}} u(t) \overline{v(t)} \mathrm{d} t
$$

We set also,

$$
H^{2}\left(X_{k}\right)=\left\{\begin{array}{c}
u \in L^{2}\left(X_{k}, \mathrm{C}\right) / u, u^{\prime} \text { exist and absolutely } \\
\text { continuous on } X_{k} \text { and } u^{\prime \prime} \in L^{2}\left(X_{k}, \mathrm{C}\right)
\end{array}\right\}
$$

Consider now the product Hilbert space $L^{2}\left(X_{1}, \mathrm{C}\right)$ $\times L^{2}\left(X_{2}, C\right)$ equipped with the inner product

$$
\langle U, V\rangle=\left\langle u_{1}, v_{1}\right\rangle_{1}+\left\langle u_{2}, v_{2}\right\rangle_{2}
$$

for all

$$
U=\left(u_{1}, u_{2}\right), V=\left(v_{1}, v_{2}\right) \in L^{2}\left(X_{1}, \mathrm{C}\right) \times L^{2}\left(X_{2}, \mathrm{C}\right)
$$

Fix now $\mathrm{H}=L^{2}\left(I_{1}, \mathrm{C}\right) \times L^{2}\left(I_{2}, \mathrm{C}\right)$ and denote its subspace $H=H^{2}\left(I_{1}\right) \times H^{2}\left(I_{2}\right)$ Let $L_{k}$ be the differential operator defined on $I_{k}$ by

$$
L_{k} u_{k}=a_{k} u_{k}^{\prime \prime}+b_{k} u_{k}^{\prime}, k=1,2
$$

where $a_{k}$ and $b_{k}$ are two real measurable functions on $I_{k}$. We make the following assumptions: For $k=1,2$ $\left(h_{1}\right): a_{k}$ is continuous and $a_{k}>0$ on $I_{k}, a_{k}^{\prime}, b_{k}$ are absolutely continuous on $I_{k}^{*}$.

$$
\begin{aligned}
& \left(h_{2}\right): a_{k}^{\prime}, a_{k}^{\prime \prime} \in L^{2}\left(I_{k}^{*}, \mathrm{C}\right), \\
& \lim _{x \rightarrow 0^{-}}\left(b_{1}-a_{1}^{\prime}\right)(x), \lim _{x \rightarrow 0^{+}}\left(b_{2}-a_{2}^{\prime}\right)(x)
\end{aligned}
$$

exist in $\mathbf{R}$ and $\left(b_{k}^{\prime}-a_{k}^{\prime \prime}\right)$ is bounded on $I_{k}^{*}$.

Let $\boldsymbol{A}_{1}$ and $\boldsymbol{A}_{2}$ two matrices in $G L_{2}(\mathrm{C})$ For $\boldsymbol{u}_{k} \in H^{2}\left(I_{k}\right)$, denote

$$
\boldsymbol{u}_{k}(x)=\left(\begin{array}{l}
u_{k}(x) \\
u_{k}^{\prime}(x)
\end{array}\right), x \in I_{k}, k=1,2
$$

The interface condition at the singular point $x=0$, is given by $\left(h_{3}\right): \Delta(0):=\boldsymbol{A}_{1} \boldsymbol{u}_{1}(0)-\boldsymbol{A}_{2} \boldsymbol{u}_{2}(0)=0$.

Note that this work can be easily generalized to degenerate matrix differential operators. Here the operator may have non-regular coefficients and may be singular at the extremities of intervals and especially at the interface point. In particular with this meaning this study is a proper extension of [9].

\section{Mixed Operator $(L, D(L))$ and its Adjoint}

In order to study the operator $L$, we introduce its Green formula. We will be able to obtain some characteristic properties. According to ([12], p. 189) the corresponding formal Lagrange adjoint expression of $L_{k}, k=1,2$ are given as

$$
L_{k}^{*} u_{k}=\left(a_{k} u_{k}\right)^{\prime \prime}-\left(b_{k} u_{k}\right)^{\prime}, k=1,2
$$

We consider the operator $(L, D(L))$ given by

$$
\begin{aligned}
& L(U)=L\left(u_{1}, u_{2}\right)=\left(L_{1} u_{1}, L_{2} u_{2}\right) \\
& \text { for } U=\left(u_{1}, u_{2}\right) \in D(L)
\end{aligned}
$$

where

$$
D(L)=\left\{U=\left(u_{1}, u_{2}\right) \in H / \Delta(0)=0, \beta_{a}=\beta_{b}=0\right\}
$$

and

$$
\beta_{a}=u_{1}^{\prime}(a)-\gamma_{a} u_{1}(a), \beta_{b}=u_{2}^{\prime}(b)-\gamma_{b} u_{2}(b)
$$

$\gamma_{a}$ and $\gamma_{b}$ are here two fixed real numbers.

It is easy to show that $(L, D(L))$ is a densely defined closed unbounded linear operator in $\mathrm{H}$ and hence has a unique adjoint (see for example Theorem 3.6 [5]).

For $U=\left(u_{1}, u_{2}\right), V=\left(v_{1}, v_{2}\right) \in D(L)$, and $a<\rho<0$ $<\sigma<b$, a simple calculation gives the Green's Formula.

$$
\begin{aligned}
& \int_{a}^{\rho}\left(a_{1} u_{1}^{\prime \prime}+b_{1} u_{1}^{\prime}\right)(x) \bar{v}_{1}(x) \mathrm{d} x \\
& +\int_{\sigma}^{b}\left(a_{2} u_{2}^{\prime \prime}+b_{2} u_{2}^{\prime}\right)(x) \bar{v}_{2}(x) \mathrm{d} x \\
& =\left[-\left(a_{1} \bar{v}_{1}\right)^{\prime} u_{1}+a_{1} \bar{v}_{1} u_{1}^{\prime}+b_{1} \bar{v}_{1} u_{1}\right]_{a}^{\rho} \\
& +\left[-\left(a_{2} \bar{v}_{2}\right)^{\prime} u_{2}+a_{2} \bar{v}_{2} u_{2}^{\prime}+b_{2} \bar{v}_{2} u_{2}\right]_{\sigma}^{b} \\
& +\int_{a}^{\rho}\left(\left(\left(a_{1} \bar{v}_{1}\right)^{\prime \prime}+\left(b_{1} \bar{v}_{1}\right)^{\prime}\right) u_{1}(x)\right) \mathrm{d} x \\
& +\int_{\sigma}^{b}\left(\left(\left(a_{2} \bar{v}_{2}\right)^{\prime \prime}+\left(b_{2} \bar{v}_{2}\right)^{\prime}\right) u_{2}(x)\right) \mathrm{d} x
\end{aligned}
$$

Using the conditions $\beta_{a}=0$ and $\beta_{b}=0$ we get,

$$
\begin{aligned}
\langle L U, V\rangle= & \left\langle L_{1} u_{1}, v_{1}\right\rangle_{1}+\left\langle L_{2} u_{2}, v_{2}\right\rangle_{2} \\
= & \left(\left(\left(\gamma_{\mathrm{b}} \mathrm{a}_{2}+\mathrm{b}_{2}-\mathrm{a}_{2}^{\prime}\right) \bar{v}_{2}-\mathrm{a}_{2} \bar{v}_{2}^{\prime}\right) u_{2}\right)(b) \\
& -\left(\left(\left(\gamma_{a} \mathrm{a}_{1}+\mathrm{b}_{1}-\mathrm{a}_{1}^{\prime}\right) \overline{v_{1}}-\mathrm{a}_{1} \vec{v}_{1}^{\prime}\right) u_{1}\right)(a) \\
& +\left\{\lim _{x \rightarrow 0^{-}} \boldsymbol{v}_{1}^{*} \boldsymbol{B}_{1} \boldsymbol{u}_{1}(x)-\lim _{x \rightarrow 0^{+}} \boldsymbol{v}_{2}^{*} \boldsymbol{B}_{2} \mathbf{u}_{2}(x)_{2}\right\} \\
& +\int_{a}^{0}\left(\left(\left(a_{1} \bar{v}_{1}\right)^{\prime \prime}+\left(b_{1} \bar{v}_{1}\right)^{\prime}\right) u_{1}\right)(x) \mathrm{d} x \\
& +\int_{0}^{b}\left(\left(\left(a_{2} \bar{v}_{2}\right)^{\prime \prime}+\left(b_{2} \bar{v}_{2}\right)^{\prime}\right) u_{2}\right)(x) \mathrm{d} x
\end{aligned}
$$

where $\boldsymbol{B}_{k}, k=1,2$ are matrix functions defined on $I_{k}^{*}$, given by

$$
\boldsymbol{B}_{k}=\left(\begin{array}{cc}
b_{k}-a_{k}^{\prime} & a_{k} \\
-a_{k} & 0
\end{array}\right)
$$

The matching interface condition $\Delta(0)=0$, and the notation $\boldsymbol{C}_{k}=\left(\boldsymbol{A}_{k}^{-1}\right)^{*}\left(\boldsymbol{B}_{k}\right)^{*}, k=1,2$, imply 


$$
\begin{aligned}
&\langle L U, V\rangle=\left\langle L_{1} u_{1}, v_{1}\right\rangle_{1}+\left\langle L_{2} u_{2}, v_{2}\right\rangle_{2} \\
&=\left(\left(\left(\gamma_{\mathrm{b}} \mathrm{a}_{2}+\mathrm{b}_{2}-\mathrm{a}_{2}^{\prime}\right) \bar{v}_{2}-\mathrm{a}_{2} \bar{v}_{2}^{\prime}\right) u_{2}\right)(b) \\
&-\left(\left(\left(\gamma_{a} \mathrm{a}_{1}+\mathrm{b}_{1}-\mathrm{a}_{1}^{\prime}\right) \bar{v}_{1}-\mathrm{a}_{1} \bar{v}_{1}^{\prime}\right) u_{1}\right)(a) \\
&+\left\{\lim _{x \rightarrow 0^{-}}\left(\left(\boldsymbol{C}_{1} \boldsymbol{v}_{1}\right)^{*} \boldsymbol{A}_{1} \boldsymbol{u}_{1}\right)(x)-\lim _{x \rightarrow 0^{+}}\left(\left(\boldsymbol{C}_{2} \boldsymbol{v}_{2}\right)^{*} \boldsymbol{A}_{2} \boldsymbol{u}_{2}\right)(x)\right\} \\
&+\int_{a}^{0}\left(\left(\left(a_{1} \bar{v}_{1}\right)^{\prime \prime}+\left(b_{1} \bar{v}_{1}\right)^{\prime}\right) u_{1}\right)(x) \mathrm{d} x \\
&+\int_{0}^{b}\left(\left(\left(a_{2} \bar{v}_{2}\right)^{\prime \prime}+\left(b_{2} \bar{v}_{2}\right)^{\prime}\right) u_{2}\right)(x) \mathrm{d} x \\
&= \beta_{b}^{*} u_{2}(b)-\beta_{a}^{*} u_{1}(a)+\left\langle u_{1}, L_{1}^{*} v_{1}\right\rangle_{1}+\left\langle u_{2}, L_{2}^{*} v_{2}\right\rangle_{2} \\
&+\left\{\lim _{x \rightarrow 0^{-}}\left(\left(\boldsymbol{C}_{1} \boldsymbol{v}_{1}\right)^{*} \boldsymbol{A}_{1} \boldsymbol{u}_{1}\right)(x)-\lim _{x \rightarrow 0^{+}}\left(\left(\boldsymbol{C}_{2} \boldsymbol{v}_{2}\right)^{*} \boldsymbol{A}_{2} \boldsymbol{u}_{2}\right)(x)\right\}
\end{aligned}
$$

where

$$
\begin{aligned}
& \beta_{a}^{*}=\left(\left(\gamma_{a} a_{1}+b_{1}-a_{1}^{\prime}\right) \bar{v}_{1}-a_{1} \vec{v}_{1}^{\prime}\right)(a), \\
& \beta_{b}^{*}=\left(\left(\gamma_{b} a_{2}+b_{2}-a_{2}^{\prime}\right) \bar{v}_{2}-a_{2} \vec{v}_{2}^{\prime}\right)(b)
\end{aligned}
$$

with these simplifications, we obtain the following result.

Proposition 3.1 Let $(L, D(L))$ be the operator defined as in (7) and (8). Then its adjoint $\left(L^{*}, D\left(L^{*}\right)\right)$ is a densely defined closed unbounded operator given by

$$
\begin{aligned}
D\left(L^{*}\right)=\{ & \left.V=\left(v_{1}, v_{2}\right) \in H / \Delta^{*}(0)=0, \beta_{a}^{*}=\beta_{b}^{*}=0\right\} \\
& L^{*}(V)=L^{*}\left(v_{1}, v_{2}\right)=\left(L_{1}^{*} v_{1}, L_{2}^{*} v_{2}\right) \\
& \text { for } V=\left(v_{1}, v_{2}\right) \in D\left(L^{*}\right)
\end{aligned}
$$

where $\Delta^{*}(0)=\lim _{x \rightarrow 0^{-}}\left(\boldsymbol{C}_{1} \boldsymbol{v}_{1}\right)(x)-\lim _{x \rightarrow 0^{+}}\left(\boldsymbol{C}_{2} \boldsymbol{v}_{2}\right)(x)$.

Proof. Let $(M, D(M))$ be the operator given by

$$
\begin{aligned}
D(M)= & \left\{V=\left(v_{1}, v_{2}\right) \in H / \Delta^{*}(0)=0, \beta_{a}^{*}=\beta_{b}^{*}=0\right\} \\
& M(V)=L^{*}\left(v_{1}, v_{2}\right)=\left(L_{1}^{*} v_{1}, L_{2}^{*} v_{2}\right) \\
& \text { for } V=\left(v_{1}, v_{2}\right) \in D(M)
\end{aligned}
$$

One has to prove that $M=L^{*}$ and $D(M)=D\left(L^{*}\right)$. From Green's formula, it follows that $D(M) \subseteq D\left(L^{*}\right)$. To show the opposite inclusion, it remains to verify that

$$
\left\langle L\left(u_{1}, u_{2}\right),\left(v_{1}, v_{2}\right)\right\rangle=\left\langle\left(u_{1}, u_{2}\right), L^{*}\left(v_{1}, v_{2}\right)\right\rangle
$$

for all $\left(u_{1}, u_{2}\right) \in D(L),\left(v_{1}, v_{2}\right) \in D\left(L^{*}\right)$. From (9) this is true if one proves that

$$
\begin{aligned}
& \beta_{b}^{*} u_{2}(b)-\beta_{a}^{*} u_{1}(a) \\
& +\left\{\lim _{x \rightarrow 0^{-}}\left(\left(\boldsymbol{C}_{1} \boldsymbol{v}_{1}\right)^{*} \boldsymbol{A}_{1} \boldsymbol{u}_{1}\right)(x)-\lim _{x \rightarrow 0^{+}}\left(\left(\boldsymbol{C}_{2} \boldsymbol{v}_{2}\right)^{*} \boldsymbol{A}_{2} \boldsymbol{u}_{2}\right)(x)\right\}=0 \\
& \text { If we choose }\left(u_{1}, u_{2}\right) \in D(L) \text { verifying } u_{1}(a)
\end{aligned}
$$

$$
\begin{aligned}
= & u_{2}(b)=0 \text {, then we get } \\
& \left\{\lim _{x \rightarrow 0^{-}}\left(\left(\boldsymbol{C}_{1} \boldsymbol{v}_{1}\right)^{*} \boldsymbol{A}_{1} \boldsymbol{u}_{1}\right)(x)-\lim _{x \rightarrow 0^{+}}\left(\left(\boldsymbol{C}_{2} \boldsymbol{v}_{2}\right)^{*} \boldsymbol{A}_{2} \boldsymbol{u}_{2}\right)(x)\right\}=0
\end{aligned}
$$

Now from Green's formula, we obtain

$$
\beta_{b}^{*} u_{2}(b)-\beta_{a}^{*} u_{1}(a)=0
$$

An appropriate choice of $\left(u_{1}, u_{2}\right) \in D(L)$, implies $\beta_{b}^{*}=\beta_{a}^{*}=0$ and $\Delta^{*}(0)=0$. This yields $D\left(L^{*}\right) \subset D(\mathrm{M})$ hence the proof is achieved.

\section{4. m -Dissipativity of $(L, D(L))$}

Recall first the definition due to Pazy [3].

Definition 4.1 A linear closed densely defined operator $(M, D(M))$ on a complex Hilbert space is called $m$-dissipative if

$$
\text { for all } u \in D(M), \mathfrak{R e}\langle M u, u\rangle \leq 0
$$

and $(\lambda-M)$ is surjective for some $\lambda>0$.

It is our aim to show, under certain assumptions on the coefficients of $L_{k}, k=1,2$, that the mixed operator is $m$-dissipative. The next technical lemma may be found in [9].

Lemma 4.1 Let $f, g$ two numerical functions of class $C^{1}$ on $[\alpha, \beta]$ such that $f$ is real then

$$
\begin{aligned}
& 2 \mathfrak{R e} \int_{\alpha}^{\beta}\left(f \bar{g} g^{\prime}\right)(x) \mathrm{d} x= \\
& f(\beta)|g|^{2}(\beta)-f(\alpha)|g|^{2}(\alpha)-\int_{\alpha}^{\beta} f^{\prime}(x)|g|^{2}(x) \mathrm{d} x
\end{aligned}
$$

In what follows, consider the following function matrices $\boldsymbol{T}_{k} \in M_{2}(\mathrm{C})$ on $I_{k}^{*}, k=1,2$ given by

$$
\boldsymbol{T}_{k}=\left(\begin{array}{cc}
b_{k}-a_{k}^{\prime} & a_{k} \\
a_{k} & 0
\end{array}\right)
$$

Theorem 4.1 Assume that the matrices $\boldsymbol{A}_{k}, k=1,2$ satisfy the condition

$$
\lim _{x \rightarrow 0^{-}}\left(\boldsymbol{A}_{1}^{-1}\right)^{*} \boldsymbol{T}_{1}(x) \boldsymbol{A}_{1}^{-1}=\lim _{x \rightarrow 0^{+}}\left(\boldsymbol{A}_{2}^{-1}\right)^{*} \boldsymbol{T}_{2}(x) \boldsymbol{A}_{2}^{-1}
$$

Then there exists a real $\lambda \geq 0$ such that the operator $((L-\lambda), D(L))$ is $m$-dissipative.

Proof. Let $U=\left(u_{1}, u_{2}\right) \in D(L)$, we have

$$
\begin{aligned}
& \langle L U, U\rangle=\left\langle L_{1} u_{1}, u_{1}\right\rangle_{1}+\left\langle L_{2} u_{2}, u_{2}\right\rangle_{2} \\
& =\left[\gamma_{b} a_{2}(b)+\frac{1}{2}\left(b_{2}(b)-a_{2}^{\prime}(b)\right)\right]\left|u_{2}(b)\right|^{2} \\
& \quad-\left[\gamma_{a} a_{1}(a)+\frac{1}{2}\left(b_{1}(a)-a_{1}^{\prime}(a)\right)\right]\left|u_{2}(a)\right|^{2} \\
& +\lim _{x \rightarrow 0^{-}}\left(a_{1} \bar{u}_{1} u_{1}^{\prime}\right)(x)-\lim _{x \rightarrow 0^{+}}\left(a_{2} \bar{u}_{2} u_{2}^{\prime}\right)(x) \\
& +\int_{a}^{0}\left(\left(b_{1}-a_{1}^{\prime}\right) \bar{u}_{1} u_{1}^{\prime}-a_{1}\left|u_{1}^{\prime}\right|^{2}\right)(x) \mathrm{d} x \\
& +\int_{0}^{b}\left(\left(b_{2}-a_{2}^{\prime}\right) \bar{u}_{2} u_{2}^{\prime}-a_{2}\left|u_{2}^{\prime}\right|^{2}\right)(x) \mathrm{d} x
\end{aligned}
$$


Then, by using Lemma 4.1, we get,

where

$$
\mathfrak{R}<\langle L U, U\rangle=S_{1}+S_{2}+S_{3}
$$

$$
\begin{aligned}
S_{1}= & {\left[\gamma_{b} a_{2}(b)+\frac{1}{2}\left(b_{2}(b)-a_{2}^{\prime}(b)\right)\right]\left|u_{2}(b)\right|^{2} } \\
& -\left[\gamma_{a} a_{1}(a)+\frac{1}{2}\left(b_{1}(a)-a_{1}^{\prime}(a)\right)\right]\left|u_{1}(a)\right|^{2} \\
= & \int_{a}^{0}\left(m_{1}\left|u_{1}\right|^{2}\right)^{\prime}(x) \mathrm{d} x+\int_{0}^{b}\left(m_{1}\left|u_{1}\right|^{2}\right)^{\prime}(x) \mathrm{d} x
\end{aligned}
$$

with

$$
\begin{aligned}
& m_{1}(x)=-\frac{x}{2 a}\left(2 \gamma_{a} a_{1}(a)+b_{1}(x)-a_{1}^{\prime}(x)\right), x \in I_{1} \\
& m_{2}(x)=\frac{x}{2 b}\left(2 \gamma_{a} a_{2}(b)+b_{2}(x)-a_{2}^{\prime}(x)\right), x \in I_{2}
\end{aligned}
$$

For $k=1,2$ and $\varepsilon>0$, we have,

$$
\begin{aligned}
& \left(m_{k}\left|u_{k}\right|^{2}\right)^{\prime}(x)=m_{k}^{\prime}(x)\left|u_{k}(x)\right|^{2}+2 m_{k}(x) \Re e\left(\overline{u_{k}(x)} u_{k}^{\prime}(x)\right) \\
& \leq\left|m_{k}^{\prime}(x)\right|\left|u_{k}(x)\right|^{2}+2\left|m_{k}(x)\right|\left|u_{k}(x)\right|\left|u_{k}^{\prime}(x)\right| \\
& \left.\leq\left|m_{k}^{\prime}(x)\right|\left|u_{k}(x)\right|^{2}+\left.\left|m_{k}(x)\right|\left|\frac{\left|u_{k}(x)\right|^{2}}{\varepsilon^{2}}+\right| \varepsilon u_{k}^{\prime}(x)\right|^{2}\right)
\end{aligned}
$$

So,

$$
\begin{gathered}
S_{1}=\sum_{k=1}^{2} \int_{I_{k}}\left(m_{k}\left|u_{k}\right|^{2}\right)^{\prime}(x) \mathrm{d} x \leq \sum_{k=1}^{2} \int_{I_{k}}\left(\left|m_{k}^{\prime}(x)\right|\left|u_{k}(x)\right|^{2}\right. \\
\left.\left.+\left|m_{k}(x)\right| \frac{\left|u_{k}(x)\right|^{2}}{\varepsilon^{2}}+\left|\varepsilon u_{k}^{\prime}(x)\right|^{2}\right)\right) \mathrm{d} x \\
S_{2}=\frac{1}{2}\left\{\lim _{x \rightarrow 0^{-}}\left(\left(b_{1}-a_{1}^{\prime}\right) u_{1} \bar{u}_{1}+2 \Re e a_{1} \bar{u}_{1} u_{1}^{\prime}\right)(x)\right. \\
\left.-\lim _{x \rightarrow 0^{+}}\left(\left(b_{2}-a_{2}^{\prime}\right) u_{2} \bar{u}_{2}+2 \Re e a_{2} \bar{u}_{2} u^{\prime}\right)(x)\right\} \\
=\frac{1}{2}\left\{\lim _{x \rightarrow 0^{-}}\left(\boldsymbol{u}_{1}^{*} \boldsymbol{T}_{1} \boldsymbol{u}_{1}\right)(x)-\lim _{x \rightarrow 0^{+}}\left(\boldsymbol{u}_{2}^{*} \boldsymbol{T}_{2} \mathbf{u}_{2}\right)(x)\right\} \\
S_{3}=-\frac{1}{2}\left\{\int_{a}^{0}\left(\left(b_{1}^{\prime}-a_{1}^{\prime \prime}\right)\left|u_{1}\right|^{2}+2 a_{1}\left|u_{1}^{\prime}\right|^{2}\right)(x) \mathrm{d} x\right. \\
+\int_{0}^{b}\left(\left(b_{2}^{\prime}-a_{2}^{\prime \prime}\right)\left|u_{2}\right|^{2}+2 a_{2}\left|u_{2}^{\prime}\right|^{2}\right)(x) \mathrm{d} x
\end{gathered}
$$

Thus we obtain,

$$
\begin{aligned}
& \Re \propto L U, U\rangle \leq-\frac{1}{2} \sum_{k=1}^{2} \int_{I_{k}}\left(\left(b_{k}^{\prime}-a_{k}^{\prime \prime}\right)(x)\right. \\
& -\left.\left(\left|m_{k}^{\prime}(x)\right|+\frac{\left|m_{k}(x)\right|^{2}}{\varepsilon^{2}}\right)|| u_{k}(x)\right|^{2} \mathrm{~d} x \\
& -\sum_{k=1}^{2} \int_{I_{k}}\left(a_{k}(x)-\varepsilon^{2}\left|m_{k}(x)\right|^{2}\right)\left|u_{k}^{\prime}(x)\right|^{2} \mathrm{~d} x \\
& +\frac{1}{2}\left\{\lim _{x \rightarrow 0^{-}}\left(\boldsymbol{u}_{1}^{*} \boldsymbol{T}_{1} \boldsymbol{u}_{1}\right)(x)-\lim _{x \rightarrow 0^{+}}\left(\boldsymbol{u}_{2}^{*} \boldsymbol{T}_{2} \boldsymbol{u}_{2}\right)(x)\right\}
\end{aligned}
$$

For sufficiently small $\varepsilon$, such that $\left(a_{k}-\varepsilon^{2}\left|m_{k}\right|^{2}\right) \geq 0$, we obtain,

$$
\begin{aligned}
\Re \mathcal{L}\langle U, U\rangle \leq & -\frac{1}{2} \sum_{k=1}^{2} \int_{I_{k}}\left(\left(b_{k}^{\prime}-a_{k}^{\prime \prime}\right)(x)\right. \\
& \left.-\left(\left|m_{k}^{\prime}(x)\right|+\frac{\left|m_{k}(x)\right|^{2}}{\varepsilon^{2}}\right)\right)\left|u_{k}(x)\right|^{2} \mathrm{~d} x \\
& +\frac{1}{2}\left\{\lim _{x \rightarrow 0^{-}}\left(\left(\boldsymbol{A}_{1} \boldsymbol{u}_{1}\right)^{*}\left(\left(\boldsymbol{A}_{1}^{-1}\right)^{*} \boldsymbol{T}_{1} \boldsymbol{A}_{1}^{-1}\right) \boldsymbol{A}_{1} \boldsymbol{u}_{1}\right)(x)\right. \\
& \left.-\lim _{x \rightarrow 0^{+}}\left(\left(\boldsymbol{A}_{2} \boldsymbol{u}_{2}\right)^{*}\left(\left(\boldsymbol{A}_{2}^{-1}\right)^{*} \boldsymbol{T}_{2} \boldsymbol{A}_{2}^{-1}\right) \boldsymbol{A}_{2} \boldsymbol{u}_{2}\right)(x)\right\} \\
= & \sum_{k=1}^{2} \int_{I_{k}}\left(\left(b_{k}^{\prime}-a_{k}^{\prime \prime}\right)(x)\right. \\
& \left.+\left(\left|m_{k}^{\prime}(x)\right|+\frac{\left|m_{k}(x)\right|^{2}}{\varepsilon^{2}}\right)\right)\left|u_{k}(x)\right|^{2} \mathrm{~d} x \\
\leq & \lambda\left(\left\|u_{1}\right\|^{2}+\left\|u_{2}\right\|^{2}\right)=\lambda\left\|\left(u_{1}, u_{2}\right)\right\|^{2}=\lambda\|U\|^{2}
\end{aligned}
$$

where,

$$
\lambda=\max _{k=1,2}\left\{\sup _{x \in I_{k}^{*}}\left(\left|b_{k}^{\prime}-a_{k}^{\prime \prime}\right|(x)+\left(\left|m_{k}^{\prime}(x)\right|+\frac{\left|m_{k}(x)\right|^{2}}{\varepsilon^{2}}\right)\right)\right\}
$$

Thus, we have shown that $(L-\lambda)$ is dissipative. For showing that $(L-\lambda)$ is $m$-dissipative, we have to show that $\left(L^{*}-\lambda\right)$ is also dissipative. The interface term vanishes, since $\left(v_{1}, v_{2}\right)$ verifies the condition,

$$
\lim _{x \rightarrow 0^{-}}\left(\left(\boldsymbol{C}_{1}^{-1}\right)^{*} \boldsymbol{T}_{1} \boldsymbol{C}_{1}^{-1} \boldsymbol{V}_{1}\right)(x)-\lim _{x \rightarrow 0^{+}}\left(\left(\boldsymbol{C}_{2}^{-1}\right)^{*} \boldsymbol{T}_{2} \boldsymbol{C}_{2}^{-1} \boldsymbol{V}_{2}\right)(x)=0,
$$

which itself is a consequence of (12). So, using same techniques as above, for all $V=\left(v_{1}, v_{2}\right) \in D\left(L^{*}\right)$, we get,

$$
\begin{aligned}
& \left\langle L^{*} V, V\right\rangle=\left\langle L_{1}^{*} v_{1}, v_{1}\right\rangle+\left\langle L_{2}^{*} v_{2}, v_{2}\right\rangle_{2} \\
& =\gamma_{b}\left(a_{2}\left|v_{2}\right|^{2}\right)(b)-\gamma_{a}\left(a_{1}\left|v_{1}\right|^{2}\right)(a)+\left\{\lim _{x \rightarrow 0^{-}}\left(\left(a_{1}^{\prime}-b_{1}\right)\left|v_{1}\right|^{2}+a_{1} v_{1}^{\prime} \bar{v}_{1}\right)(x)-\lim _{x \rightarrow 0^{+}}\left(\left(a_{2}^{\prime}-b_{2}\right)\left|v_{2}\right|^{2}+a_{2} v_{2}^{\prime} \bar{v}_{2}\right)(x)\right\} \\
& +\int_{a}^{0}\left(\left(b_{1}-a_{1}^{\prime}\right) v_{1} \vec{v}_{1}^{\prime}-a_{1}\left|v_{1}^{\prime}\right|^{2}\right)(x) \mathrm{d} x+\int_{0}^{b}\left(\left(b_{2}-a_{2}^{\prime}\right) v_{2} \vec{v}_{2}^{\prime}-a_{2}\left|v_{2}^{\prime}\right|^{2}\right)(x) \mathrm{d} x
\end{aligned}
$$


Then one has, $\Re \in\left\langle L^{*} V, V\right\rangle \leq \frac{1}{2} \sum_{k=1}^{2} \int_{I_{k}}\left(\left(b_{k}^{\prime}-a_{k}^{\prime \prime}\right)(x)\right.$

$$
\begin{aligned}
& \left.+\left(\left|m_{k}^{\prime}(x)\right|+\left|m_{k}(x)\right|^{2} / \varepsilon^{2}\right)\right)\left|v_{k}(x)\right|^{2} \mathrm{~d} x \\
& \leq \lambda\left(\left\|v_{1}\right\|^{2}+\left\|v_{2}\right\|^{2}\right)=\lambda\left\|\left(v_{1}, v_{2}\right)\right\|^{2}=\lambda\|V\|^{2}
\end{aligned}
$$

This implies that both $(L-\lambda)$ and $\left(L^{*}-\lambda\right)$ are dissipative, thus $(L-\lambda)$ is $m$-dissipative and hence the theorem is proved.

\section{Analyticity of the Semigroup Generated by $(L, D(L))$}

The purpose of this section is to prove the analyticity of the semigroup generated by $(L, D(L))$. For This goal we impose some additional conditions on the matrices $A_{k}, k=1,2$. In the following we recall a theorem due to Fattorini [11].

Theorem 5.1 Let $(A, D(A))$ be a densely defined operator in a Hilbert space such that for any $u \in D(A)$ :

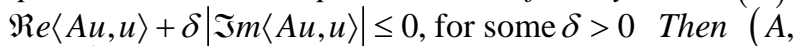
$D(A)$ ) generates an analytic semigroup of contractions.

With the help of Theorem 5.1, we will establish our main result.

Theorem 5.2 Assume that the matrices $\boldsymbol{B}_{k}$ and $\boldsymbol{T}_{k}, k=1,2$ defined respectively in sections 3 and 4 , satisfy the conditions

$$
\begin{aligned}
& \lim _{x \rightarrow 0^{-}}\left(\boldsymbol{A}_{1}^{-1}\right)^{*} \boldsymbol{B}_{1}(x) \boldsymbol{A}_{1}^{-1}=\lim _{x \rightarrow 0^{+}}\left(\boldsymbol{A}_{2}^{-1}\right)^{*} \boldsymbol{B}_{2}(x) \boldsymbol{A}_{2}^{-1} \\
& \lim _{x \rightarrow 0^{-}}\left(\boldsymbol{A}_{1}^{-1}\right)^{*} \boldsymbol{T}_{1}(x) \boldsymbol{A}_{1}^{-1}=\lim _{x \rightarrow 0^{+}}\left(\boldsymbol{A}_{2}^{-1}\right)^{*} \boldsymbol{T}_{2}(x) \boldsymbol{A}_{2}^{-1}
\end{aligned}
$$

Then the operator $(L, D(L))$ generates an analytic semigroup of contractions.

Proof. Since the operator $(L, D(L))$ is densely defined, then from Theorem 5.1, to show that it generates an analytic semigroup, it suffices to verify that $\operatorname{Re}\langle A u, u\rangle+\delta|\operatorname{Im}\langle A u, u\rangle| \leq 0$, for $A=L-\rho I$ and for some $\rho>0$ and $\delta>0$.

This is equivalent to show that $\mathfrak{R} e\langle L u, u\rangle$ $+\delta|\Im m\langle L u, u\rangle| \leq \rho\|u\|^{2}$.

Holds for all $u \in D(L)$. Using the identity

$$
\begin{aligned}
\langle L U, U\rangle= & {\left[\gamma_{b} a_{2}(b)+(1 / 2)\left(b_{2}(b)-a_{2}^{\prime}(b)\right)\right]\left|u_{2}(b)\right|^{2} } \\
& -\left[\gamma_{a} a_{1}(a)+(1 / 2)\left(b_{1}(a)-a_{1}^{\prime}(a)\right)\right]\left|u_{1}(a)\right|^{2} \\
& +\lim _{x \rightarrow 0^{-}}\left(a_{1} \bar{u}_{1} u_{1}^{\prime}\right)(x)-\lim _{x \rightarrow 0^{+}}\left(a_{2} \bar{u}_{2} u_{2}^{\prime}\right)(x) \\
& +\int_{a}^{0}\left(\left(b_{1}-a_{1}^{\prime}\right) \bar{u}_{1} u_{1}^{\prime}-a_{1}\left|u_{1}^{\prime}\right|^{2}\right)(x) \mathrm{d} x \\
& +\int_{0}^{b}\left(\left(b_{2}-a_{2}^{\prime}\right) \bar{u}_{2} u_{2}^{\prime}-a_{2}\left|u_{2}^{\prime}\right|^{2}\right)(x) \mathrm{d} x
\end{aligned}
$$

then

$$
\begin{aligned}
& \Im \mathrm{m}\langle L U, U\rangle=\mathfrak{I m}\left\{\lim _{x \rightarrow 0^{-}}\left(a_{1} \bar{u}_{1} u_{1}^{\prime}\right)(x)-\lim _{x \rightarrow 0^{+}}\left(a_{2} \bar{u}_{2} u_{2}^{\prime}\right)(x)\right\} \\
& +\mathfrak{I m}\left\{\int_{a}^{0}\left(\left(b_{1}-a_{1}^{\prime}\right) \bar{u}_{1} u_{1}^{\prime}\right)(x) \mathrm{d} x+\int_{0}^{b}\left(\left(b_{2}-a_{2}^{\prime}\right) \bar{u}_{2} u_{2}^{\prime}\right)(x) \mathrm{d} x\right\} \\
& =J_{1}+J_{2}
\end{aligned}
$$

where

$$
J_{1}=\mathfrak{I m}\left\{\lim _{x \rightarrow 0^{-}}\left(a_{1} \bar{u}_{1} u_{1}^{\prime}\right)(x)-\lim _{x \rightarrow 0^{+}}\left(a_{2} \bar{u}_{2} u_{2}^{\prime}\right)(x)\right\}
$$

and

$J_{2}=$

$$
\text { Im }\left\{\int_{a}^{0}\left(\left(b_{1}-a_{1}^{\prime}\right) \bar{u}_{1} u_{1}^{\prime}\right)(x) \mathrm{d} x+\int_{0}^{b}\left(\left(b_{2}-a_{2}^{\prime}\right) \bar{u}_{2} u_{2}^{\prime}\right)(x) \mathrm{d} x\right\}
$$

Using the relation $2 \mathfrak{I m}(\lambda)=\mathfrak{I m}(\lambda-\bar{\lambda})$ for all $\lambda \in \square$, we deduce the expression

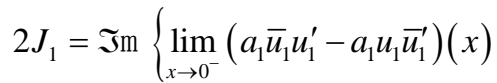

$$
\begin{aligned}
& \left.-\lim _{x \rightarrow 0^{+}}\left(a_{2} \bar{u}_{2} u_{2}^{\prime}-a_{2} u_{2} \bar{u}_{2}^{\prime}\right)(x)\right\} \\
& =\mathfrak{I m}\left\{\lim _{x \rightarrow 0^{-}}\left(\left(\boldsymbol{A}_{1} \boldsymbol{u}_{1}\right)^{*}\left(\boldsymbol{A}_{1}^{-1}\right)^{*} \boldsymbol{B}_{1} \boldsymbol{A}_{1}^{-1}\left(\boldsymbol{A}_{1} \boldsymbol{u}_{1}\right)\right)(x)\right. \\
& \left.-\lim _{x \rightarrow 0^{+}}\left(\left(\boldsymbol{A}_{2} \boldsymbol{u}_{2}\right)^{*}\left(\boldsymbol{A}_{2}^{-1}\right)^{*} \boldsymbol{B}_{2} \boldsymbol{A}_{2}^{-1}\left(\boldsymbol{A}_{1} \boldsymbol{u}_{2}\right)\right)(x)\right\}
\end{aligned}
$$

Under the assumption (13) and the interface condition, we get

$$
\begin{aligned}
& 2 J_{1}=\mathfrak{I m}\left\{\left(\lim _{x \rightarrow 0^{-}}\left(\boldsymbol{A}_{1} \boldsymbol{u}_{1}\right)^{*}(x)\right)\right. \\
& \left(\lim _{x \rightarrow 0^{-}}\left(\left(\boldsymbol{A}_{1}^{-1}\right)^{*} \boldsymbol{B}_{1} \boldsymbol{A}_{1}^{-1}\right)(x)-\lim _{x \rightarrow 0^{+}}\left(\left(\boldsymbol{A}_{2}^{-1}\right)^{*} \boldsymbol{B}_{2} \boldsymbol{A}_{2}^{-1}\right)(x)\right) \\
& \left.\left(\lim _{x \rightarrow 0^{+}}\left(\boldsymbol{A}_{2} \boldsymbol{u}_{2}\right)(x)\right)\right\}=0
\end{aligned}
$$

We have also, for sufficiently small $\varepsilon$,

$$
\begin{aligned}
& \left|J_{2}\right|=\mid \mathfrak{I m}\left\{\int_{a}^{0}\left(\left(b_{1}-a_{1}^{\prime}\right) \bar{u}_{1} u_{1}^{\prime}\right)(x) \mathrm{d} x\right. \\
& \left.+\int_{0}^{b}\left(\left(b_{2}-a_{2}^{\prime}\right) \bar{u}_{2} u_{2}^{\prime}\right)(x) \mathrm{d} x\right\} \mid
\end{aligned}
$$

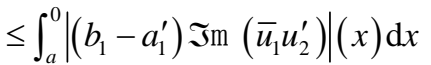

$$
\begin{aligned}
& +\int_{0}^{b}\left|\left(b_{2}-a_{2}^{\prime}\right) \mathfrak{I m}\left(\bar{u}_{2} u_{2}^{\prime}\right)\right|(x) \mathrm{d} x \\
& \leq \int_{a}^{0}\left(\left|\left(b_{1}-a_{1}^{\prime}\right)\right| u_{1} u_{1}^{\prime} \mid\right)(x) \mathrm{d} x \\
& +\int_{0}^{b}\left(\left|\left(b_{2}-a_{2}^{\prime}\right)\right|\left|u_{2} u_{2}^{\prime}\right|\right)(x) \mathrm{d} x \\
& \leq \int_{a}^{0}\left(\left|\left(b_{1}-a_{1}^{\prime}\right)\right|\left(\varepsilon^{-2}\left|u_{1}\right|^{2}+\varepsilon^{2}\left|u_{1}^{\prime}\right|^{2}\right)\right)(x) \mathrm{d} x \\
& +\int_{0}^{b}\left(\left|\left(b_{2}-a_{2}^{\prime}\right)\right|\left(\varepsilon^{-2}\left|u_{2}\right|^{2}+\varepsilon^{2}\left|u_{2}^{\prime}\right|^{2}\right)\right)(x) \mathrm{d} x
\end{aligned}
$$

It follows that, for $\delta>0$, we have 


$$
\begin{aligned}
& \mathfrak{R e}\langle L u, u\rangle+\delta|\mathfrak{I} m\langle L u, u\rangle| \\
& \leq \sum_{k=1}^{2} \int_{I_{k}}\left\{\left|b_{k}^{\prime}-a^{\prime \prime}\right|(x)+\left(\left|m_{k}^{\prime}(x)\right|+\frac{\left|m_{k}(x)\right|^{2}}{\varepsilon^{2}}\right)\right\}\left|u_{k}(x)\right|^{2} \mathrm{~d} x \\
& \quad+\frac{\delta}{2 \varepsilon^{2}} \sum_{k=1}^{2} \int_{I_{k}}\left\{\left|b_{k}-a_{k}^{\prime}\right|\left|u_{k}\right|^{2}(x) \mathrm{d} x\right\} \\
& +\frac{\delta}{2} \sum_{k=1}^{2} \int_{I_{k}}\left\{\left(\left|\varepsilon^{2}\right|\left(b_{k}-a_{k}^{\prime}+\left|m_{k}\right|\right)-a_{k}\right)\left|u_{k}^{\prime}\right|^{2}\right\}(x) \mathrm{d} x \\
& \leq \rho\|u\|^{2}
\end{aligned}
$$

where

$$
\begin{aligned}
\rho= & \max _{k=1,2}\left\{\operatorname { s u p } _ { x \in I _ { k } ^ { * } } \left\{\left|b_{k}^{\prime}-a_{k}^{\prime \prime}\right|+\left|m_{k}^{\prime}\right|\right.\right. \\
& \left.\left.+\varepsilon^{-2}\left|m_{k}\right|^{2}+\varepsilon^{-2} \frac{\delta}{2}\left|b_{k}-a_{k}^{\prime}\right|\right\}(x)\right\}
\end{aligned}
$$

Thus the proof is achieved and the result of the Theorem is obtained.

Corollary 5.1 The operator $(L+B, D(L))$ generates an analytic semigroup for all $L$-Bounded operators $B$. In particular the result remains true if we choose $B=\left(R_{1}, R_{2}\right)$ defined on $\mathrm{H}$ by $R_{k} u_{k}=c_{k} u_{k}, k=1,2$, where $c_{k}$ is a piecewise continuous function on $I_{k}$.

For more detail in perturbation theory of linear operators we refer to [7] and [13].

In the following an example is given to demonstrate the effectiveness of our results.

Example 5.1 Let $I_{1}=[-1,0]$ and $I_{2}=[0,1]$, and consider the following differential system

$$
\left\{\begin{array}{cc}
\frac{\partial u_{1}}{\partial t}=a_{1}(x) \frac{\partial^{2} u_{1}}{\partial^{2} x}+b_{1}(x) \frac{\partial u_{1}}{\partial x}, & x \in I_{1}^{*} \\
\frac{\partial u_{2}}{\partial t}= & a_{2}(x) \frac{\partial^{2} u_{2}}{\partial^{2} x}+b_{2}(x) \frac{\partial u_{2}}{\partial x}, \quad x \in I_{2}^{*} \\
& \left(u_{1}, u_{2}\right)(0)=u_{0}
\end{array}\right.
$$

where $a_{k}, b_{k}, k=1,2$ are real functions verifying the previous assumptions $h_{1}$ and $h_{2}$.

The interface condition at $x=0$ is such that

$$
a \frac{\partial u_{1}}{\partial x}(0, t)=b \frac{\partial u_{2}}{\partial x}(0, t), u_{1}(0, t)=-u_{2}(0, t) .
$$

The end points conditions are taken to be

$$
\begin{aligned}
& \beta_{1}=\frac{\partial u_{1}}{\partial x}(-1, t)-\alpha_{1} u_{1}(-1, t)=0, \\
& \beta_{2}=\frac{\partial u_{2}}{\partial x}(1, t)-\alpha_{2} u_{2}(1, t)=0
\end{aligned}
$$

for some real constants $a, b, c, \alpha_{1}$ and $\alpha_{2}$.
The operator $(S, D(S))$ is as follows

$$
S=\left(S_{1}, S_{2}\right), S_{k} u_{k}=a_{k} u_{k}^{\prime \prime}+b_{k} u_{k}^{\prime}, k=1,2
$$

and

$$
\begin{gathered}
D(S)=\left\{U=\left(u_{1}, u_{2}\right) \in H \mid \boldsymbol{A}_{1} \boldsymbol{u}_{1}(0)=\boldsymbol{A}_{2} \boldsymbol{u}_{2}(0),\right. \\
\left.\beta_{1}=\beta_{2}=0\right\}
\end{gathered}
$$

where $\boldsymbol{A}_{1}=\left(\begin{array}{ll}0 & a \\ c & 0\end{array}\right)$ and $\boldsymbol{A}_{2}=\left(\begin{array}{cc}0 & b \\ -c & 0\end{array}\right)$.

Then it is easily to verify that the conditions of Theorem 5.2 are fulfilled for the operator $(S, D(S))$ if $a_{k}(0)>0$ for $k=1,2, \lim _{\left(0 \rightarrow 0^{-}\right.}\left(b_{1}-a_{1}^{\prime}\right)(x)=\lim _{x \rightarrow 0^{+}}\left(b_{2}-a_{2}^{\prime}\right)$ $(x)$ and $b a_{1}(0)+a a_{2}(\sigma) \stackrel{0^{-}}{=} 0$

Then for all $u_{0} \in H$, the above evolution partial differential system has a unique solution which is analytic in time for $t>0$.

The following functions are a concrete example for the above system.

$$
\begin{aligned}
& a_{1}(x)=\int_{0}^{x} t^{2} \sin \left(\frac{1}{t}\right) \mathrm{d} t+\gamma_{1}, b_{1}(x)=x, \gamma_{1}>\frac{1}{3} \\
& a_{2}(x)=1-x^{2-\gamma_{2}} \log (x), b_{2}=a_{2}^{\prime}, \gamma_{2}<\frac{1}{2}
\end{aligned}
$$

with $b \gamma_{1}+a=0$.

\section{References}

[1] K. Ito and F. Kappel, "Evolution Equations and Approximations," Series on Advances in Mathematics for Applied Sciences, World Scientific Publishing Company, River Edge, Vol. 61, 2002.

[2] C. A. Boyes, "Acoustic Waveguides," Application to Oceanic Sciences, Wiley, New York, 1984.

[3] A. Pazy, "Semigroups of Linear Operators an Applications to Partial Differential Equations," Applied Math Sciences, Springer, New York, Vol. 44, 1983.

[4] N. H. Mahmoud, "Partial Differential Equations with Matricial Coefficients and Generalized Translation Operators," Transactions of the American Mathematical Society, Vol. 352, No. 8, 2000, pp. 3687-3706.

[5] N. H. Mahmoud, "Heat Equations Associated with Matrix Singular Differential Operators and Spectral Theory," Integral Transforms and Special Functions, Vol. 15, No. 3, 2004. pp. 251-266. doi:10.1080/10652460310001600591

[6] J. Weidmann, "Spectral Theory of Ordinary Differential Operators," Lecture Notes in Mathematics, Springer, Berlin, Vol. 1258, 1987.

[7] K. J. Engel and R. Nagel, "One-Parameter Semigroups for Linear Evolution Equations,” Springer-Verlag, New York, 2000.

[8] R. Nagel, "One-Parameter Semigroups of Positive Op- 
erators,” Lecture Notes in Mathematics, Springer- Verlag, Berlin, Vol. 1184, 1986.

[9] A. Saddi and O. A. Mahmoud Sid Ahmed, “Analyticity of Semigroups Generated by a Class of Differential Operators with Matrix Coefficients and Interface," Semigroup Forum, Vol. 71, No. 1, 2005, pp. 1-17. doi:10.1007/s00233-004-0173-6

[10] T. G. Bhaskar and R. Kumar, "Analyticity of Semigroup Generated by a Class of Differential Operators with In- terface,” Nonlinear Analysis, Vol. 39, No. 6, 2000, pp. 779-791. doi:10.1016/S0362-546X(98)00237-5

[11] H. O. Fattorini, “The Cauchy Problem,” Addison Wesley, Massachusetts, Vol. 18, 1983.

[12] H. Chebli, “Analyse Hilbertienne,” Centre de Publication Universitaire, Tunis, 2001.

[13] T. Kato, "Perturbation Theory for Linear Operators," Springer-Verlag, Berlin, 1966. 